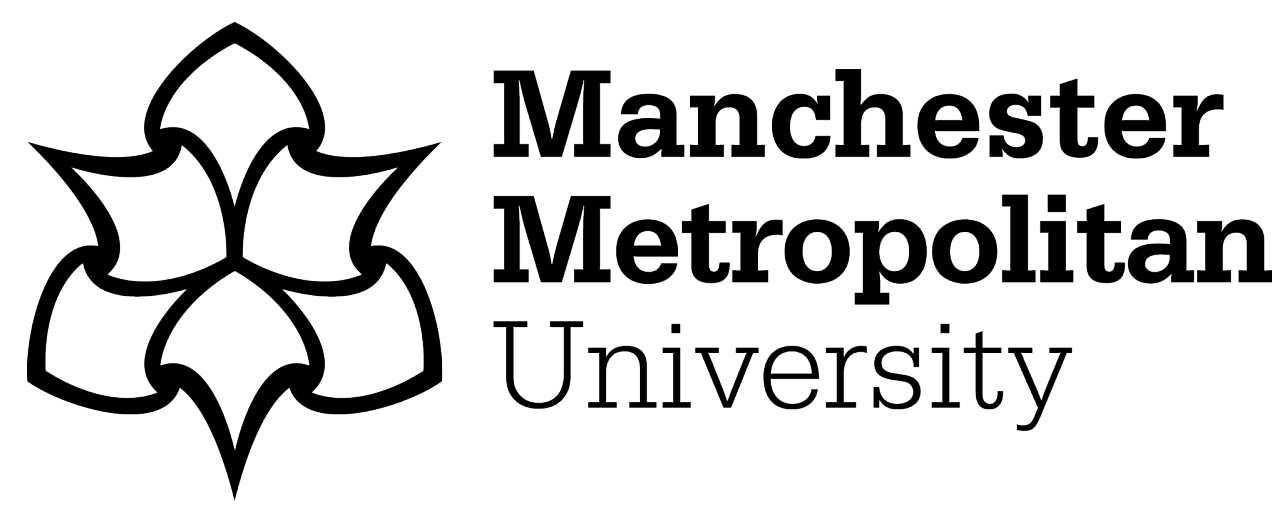

Shah, Syed Aziz ORCID logoORCID: https://orcid.org/0000-0003-20521121, Fan, Dou, Ren, Aifeng, Zhao, Nan, Yang, Xiaodong and Tanoli, Shujaat Ali Khan (2020) Seizure episodes detection via smart medical sensing system. Journal of Ambient Intelligence and Humanized Computing, 11 (11). pp. 4363-4375. ISSN 1868-5137

Downloaded from: https://e-space.mmu.ac.uk/624438/

Version: Accepted Version

Publisher: Springer

DOI: https://doi.org/10.1007/s12652-018-1142-3

Please cite the published version 


\title{
Seizure episodes detection via smart medical sensing system
}

\author{
Syed Aziz Shah ${ }^{1,2} \cdot$ Dou Fan $^{1} \cdot$ Aifeng Ren ${ }^{1} \cdot$ Nan Zhao $^{1} \cdot$ Xiaodong Yang $^{1}$ (D) Shujaat Ali Khan Tanoli ${ }^{3}$
}

Received: 28 August 2018 / Accepted: 15 November 2018

(c) Springer-Verlag GmbH Germany, part of Springer Nature 2018

\begin{abstract}
Cyber-physical systems (CPS) consist of seamless network of sensors and actuators integrated with physical processes related to human activities. The CPS exploits sensors and actuators to monitor and control different physical process that can affect the computations of the devices. This paper presents the monitoring of physical activities exploiting wireless devices as sensors used in medical cyber-physical systems. Patients undergoing epileptic seizures experience involuntary body movements such as jerking, muscle twitching, falling, and convulsions. The proposed method exploits S-Band sensing used in medical CPS that leverage wireless devices such as omni-directional antenna at the transmitter side, four-beam patch antenna at the receiver side, RF signal generator and vector signal analyzer that perform signal conditioning by providing amplitude and raw phase data. The method uses wireless monitoring and recording system for measurement and classification of a clinical condition (epileptic seizures) versus normal daily routine activities. The data acquired that are perturbations of the radio signal is analyzed as amplitude, phase information, and statistical models. Extracting the statistical features, we leverage various machine learning algorithms such as support vector machine, random forest, and K-nearest neighbor that classify the data to differentiate patient's various activities such as press-ups, walking, sitting, squatting, and seizure episodes. The performance parameters used in three machine learning algorithms are accuracy, precision, recall, Cohen's Kappa coefficient, and F-measure. The values obtained using five performance parameters provide the accuracy of more than $90 \%$.
\end{abstract}

Keywords Smart medical sensing system $\cdot$ Machine learning $\cdot$ Internet of things

\section{Introduction}

Cyber-physical systems (CPS) and smart systems consist of seamless network of sensors and actuators integrated with physical processes (Lotfi et al. 2012), related to human activities. The CPS exploits sensors and actuators to monitor and control different physical processes that can affect the computations of the devices. The various physical processes can be temperature monitoring and control, human intrusion detection and alarm notification, surveillance, different disease monitoring in medical healthcare, etc. This

Xiaodong Yang

xdyang@xidian.edu.cn

1 School of Electronic Engineering, Xidian University, Xi' an 710071, Shaanxi, China

2 School of Engineering, University of Glasgow, Glasgow G12 8QQ, UK

3 Department of Electrical Engineering, COMSATS Institute of information Technology, Attock, Pakistan paper presents different wireless devices such as omnidirectional antenna, four-beam patch antenna, RF generator, and vector network analyzer used as sensors which is part to monitor particular physical condition experienced by patient suffering from epilepsy disease. The seamless integration of particular physical devices for monitoring physical activities in medical healthcare is known as medical CPS and the sensing technique adopted is termed as S-Band sensing since it operates in $2 \mathrm{GHz}$ to $4 \mathrm{GHz}$ (Table 1 ).

Healthy brain and healthy body give rise to productive and prosperous societies. With healthcare taking a global momentum, medical conditions such as epilepsy disease (ED) is increasingly affecting the performance of individuals. The cause of epilepsy is not clear either genetic or due to traumatic events. It is known to affect brain functioning and can cause secondary infection hampering their everyday activity. Epilepsy is an incessant neurological disorder that patients have recurring incidents that is unpredictable. Seizures that are intermittent within a short period affect brain cells or neurons with sudden activity (Dilber and Kaur 2016). These 
Table 1 Comparison of different human recognition methods

\begin{tabular}{lllll}
\hline ID & References & Methodology & Advantage & Discussion \\
\hline 1 & Ben-Arie et al. (2012) & Multiple cameras & Activity detection using few video frames & Light-dependent \\
2 & Song et al. (2010) & Dist. camera networks & Off-the-shelf cameras & Light-dependent \\
3 & Tao et al. (2014) & Wearable inertial sensors & Low computational cost & Deployment of sensors on body \\
4 & Abdelnasser et al. (2014) & RSSI & Based on commercially available wireless devices & Path-loss characterization \\
5 & Sigg et al. (2014) & RSSI & Based on commercially available wireless devices & Path-loss characterization \\
\hline
\end{tabular}

active neurons cause synchronous transmission of electrical signals. The electrical signal is an ion that travels in and out of a neuron through protein channels. The neurotransmitter receptors that regulate the activity of ion channels to receive electrical signals are known as excitatory neurotransmitters while those responsible for stopping the electrical messages are termed as inhibitory neurotransmitters (Pedram et al. 2015). When epileptic patients experience a seizure, the clusters of neurons become briefly dysfunctional. As a result, the brain transmits millions of excitatory electrical signals over a period of time. The excitatory electrical signals cause a transmission of messages by a large group of neurons. This simultaneous message transmission can cause small and large-scale body movements including falling, losing balance, muscle twitching, jerking, losing conscious and vomiting. These outward signs are, however, subjective experiences that may vary from person to person and depend on the affected area of the brain due to the seizure. Therefore, constant monitoring of patients suffering from epileptic seizures is necessary at home or hospitals to ensure timely intervention that can reduce the risk of a serious injury.

With the recent growth in wireless technology, applications of RF signals have increased from merely a communication link to that of a wireless sensing platform (Yang et al. 2018a, b, c). Transmitted RF signal propagates through various paths when an obstacle such as a human subject blocks the direct path. Different body postures would perturb the signal changes as seen in the received signal. By analyzing the characteristics of the received signal, human subject's behavior and postures such as sleeping positions, falls or gestures can be monitored and any changes can be identified effectively.

The proposed method uses the S-Band sensing technique for acquiring and classifying the data. The signal variances of wireless channel information caused by the patient seizure episodes over a period of time are analyzed. The method extracts the behavioral pattern from the amplitude and phase information of wireless channel information. The system offers non-invasive implementation where no device is deployed on subject's body. With the system having high directivity providing high accuracy in classifying various human activities such as sit-stand, walking into the room, press-ups, sitting on a chair and seizures. For classifying various activities, we use three machine learning algorithms such as support vector machine (SVM), K-nearest neighbor (KNN) and Random Forest (RF). In the subsequent sections, we discuss the proposed model for feature extraction and classification method.

\section{Related work}

Human activity is represented as a set of 18-dimensional feature vectors, these define the pose angles and velocities of various body parts during movement. In a human activity monitoring work, (Ben et al. 2002; Castillo et al. 2017) introduced a vision-based recognition system. Another activity based monitoring system was introduced by Tambe and Gajre (2018) which was based cluster analysis of mobile healthcare application for prediction exploiting physiological data. An efficient and robust system based on distributed camera networks for classifying various human activities was also introduced (Song et al. 2010; Alemdar and Ersoy 2017). Since the solutions are imagebased, the lighting conditions, orientations, and filtering for processing are exhaustive. Accordingly, capturing of images is extremely important for differentiation of the features. This dependency is factored into accuracy rate that needs to be consistent On the other hand, the wearable inertial sensors are used for activity recognition for certain applications (Azimi et al. 2017) The problem with a system based on wearable inertial sensors is that the sensing components must be deployed on subject's body that might cause uneasiness for the user in long-term usages. Human activity recognition systems based on received signal strength indicator (RSSI) characterized only path-loss (Abdelnasser et al. 2015; Sigg et al. 2014), report limited identification accuracy.

The design of the signal processing in signal denoising, gestures as a set of features are extracted and performance is determined by the accuracy rate of gesture detection. The challenges in this wireless system include noisy RSSI values due to multipath interference and other electromagnetic noise that increase the false positive rates (Shah et al. 2016). 


\section{System model}

This section presents the system model and data acquisition using S-Band sensing, phase calibration, receiving antenna model and data classification methods.

\subsection{S-band sensing technique for data acquisition}

The proposed system is based on S-Band technique that leverages wireless devices such as RF generator connected to an omni-directional operating at $2.4 \mathrm{GHz}$. The RF generator transmits 30 frequency carriers, simultaneously that contain the variances of amplitude and phase information. A wireless signal upon being reflected, refracted, shadowed and affected by multipath propagation is as it encounters an obstacle in the path. The received signal can be represented as:

$\mathbf{Y}=\mathrm{WCI} \times \mathbf{X}+\mathbf{N}$

here, $X$ and $Y$ represent the transmitted and received signals, respectively. $N$ is the channel noise and $W C I$ denotes the channel frequency response (CFR).

The 30 frequency carriers received using four-beam patch antenna connected to vector network analyzer is termed as one WCI packet containing amplitude and phase information. This WCI information can be expressed as:

$W C I_{n}=\left|W C I_{n}\right| e^{j<\theta}$

$W C I_{n}$ denotes the channel frequency response of $n$th frequency carrier, $\left|W C I_{n}\right|$ and $\angle W C I_{n}$ are the amplitude and raw $W C I$ phase information, respectively.

\subsection{WCI phase calibration}

The raw WCI data obtained using four-beam patch antenna connected to vector network analyzer working as the receiver is extremely random and inapplicable for detecting epileptic seizure episodes. An accurate detection of the human subject's behavior and the epileptic seizure episodes require amplitude as well as the phase information. This study, therefore, uses calibration to remove the phase offset through a linear transformation to extract useful or calibrated phase information out of the raw WCI data received using S-Band sensing technique.

The WCI raw phase information for $n$th frequency carrier can be expressed as:

$\varphi_{i}^{\prime}=\varphi_{n}-2 \pi \frac{L_{i}}{K} \partial+\sigma$

$\varphi_{n}$ carries the raw WCI phase information, ddenotes the offset timing primarily responsible for phase error and $\sigma$ denotes the unknown phase offset. $L_{\mathrm{i}}$ is the subcarrier number of the $i$ th frequency carrier. Useful phase information using vector network analyzer solely is infeasible due to the presence of unknown terms in Eq. 3. To extract the calibrated phase information from raw WCI data, a linear transformation is applied as discussed (Yang et al. 2018a, b, c). Parameters $Q$ and $R$ are introduced in order to remove $\partial$ and $\sigma$.

\subsection{Four-beam patch antenna}

The perturbation and interference experienced by an RF signal in the indoor environment need to be suppressed to improve the efficiency of the system and detection of the seizure signals. To improve the perturbation during seizure we consider directional antenna (four beam patch antenna) (Giorgetti et al. 2007) operating in the range of $2.4 \mathrm{GHz}$. This antenna offers high levels of directivity and suppresses interference due to multipath fading effectively (Giorgetti et al. 2007).

\subsection{Data classification}

The data received using S-Band sensing technique is classified using different machine learning algorithms such as SVM, KNN, and RF to differentiate the seizure episodes of the epileptic patient from other human activities such as sit-stand, walking inside the room, press-ups, sitting on a chair with body movements. The three algorithms used in this paper are discussed as follows.

\subsubsection{Support vector machine}

SVM is a statistical model for classification and mapping of the new data set that is aimed at automation. A generalized identification and association of new randomly chosen data samples with a particular class also rely on our training dataset. From the raw data, we extract ten features that are tightly bound to the amplitude of sensed signals. The training and testing data are represented as the following features as listed in Table 2. Considering $c$, the input vector and $P$ the total number of support vectors both characterize the training data. The organization of data is based on measurements per activity for a finite number of packets. The second level of data points is a set of features that form a "hyperplane" defining an optimal set of data points as boundaries.

Table 2 shows the list of features that are set up as a measure of comparison. For example: root mean square of any signal signifies the steady value, the peak to peak varies thus helping us differentiate the signals and variations of the perturbations of the RF. Similarly, skewness gives a measure of symmetry while kurtosis for disproportionality measure for a given distribution set of samples. 
Table 2 Feature set extracted from the raw data

\begin{tabular}{|c|c|c|c|c|}
\hline $\begin{array}{l}\text { Root mean square } F_{R M S} \\
\sqrt{\frac{1}{P} \sum_{i=1}^{P} c_{i}^{2}}\end{array}$ & Crest factor, $F_{C F} \frac{\max \left(\left|c_{i}\right|\right)}{F_{R M S}}$ & Marginal factor, $F_{M F} \frac{\max |c|}{F_{S R A}}$ & $\begin{array}{l}\text { Skewness value, } F_{S V} \\
\frac{1}{P} \sum_{i=1}^{P}\left[\frac{\left[c_{i}-\mu_{c}\right]}{\sigma}\right]^{3}\end{array}$ & $\begin{array}{l}\text { The square root of } \\
\text { amplitude, } F_{S R A} \\
{\left[\frac{1}{P} \sum_{i=1}^{P} \sqrt{\left|c_{i}\right|}\right]^{2}}\end{array}$ \\
\hline Impact factor $F_{S F} \frac{\max (|c|)}{\frac{1}{P} \sum_{i=1}^{P}\left|c_{i}\right|}$ & Mean value, $F_{M V} \frac{1}{P} \sum_{i=1}^{P} c_{i}$ & $\begin{array}{l}\text { Peak-to-peak value } F_{P P V} \\
\max (c)-\min (c)\end{array}$ & $\begin{array}{l}\text { Kurtosis value } F_{K V} \\
\frac{1}{P} \sum_{i=1}^{P}\left[\frac{\left[c_{i}-\mu_{c}\right]}{\sigma}\right]^{4}\end{array}$ & $\begin{array}{l}\text { Standard deviation, } F_{S T D} \\
\sqrt{\frac{1}{P} \sum_{i=1}^{P}\left(c_{i}-\mu_{c}\right)^{2}}\end{array}$ \\
\hline
\end{tabular}

SVM is a binary classifier developed for nonlinear boundary problems (Yang et al. 2018a, b, c) and based on supervised learning method that uses kernel technique. A hyperplane is drawn that acts as a decision boundary for classifying two datasets. The data points closest to the hyperplane that separate the hyperplane are known as support vectors. The optimum hyperplane can be obtained as:

$w^{T} c+x=0$

where $w$ represents the weight vector, $c$ is the input vector and $x$ is the bias. The support vector representing each class can be written as:

$w^{T} c+x=+1 \quad$ when $\quad g_{i}=+1$

$w^{T} c+x=-1 \quad$ when $g_{i}=-1$

here $g_{i}=+1, g_{i}=-1$ represent class A and class B respectively. The optimum hyperplane for training samples can be expressed as:

$\min \vartheta(w)=\frac{1}{2} w^{T} w$

$g_{i}\left(w^{T} c_{i}+x\right) 1, \forall i=1,2,3 \ldots$

The final decision function is denoted as:

$f(c)=\operatorname{sign}\left[\sum_{i=1}^{P} \alpha_{i, o}\left(c^{T} c_{i}\right)+x\right]$

where $c$ indicates the input vector that will be classified, $P$ is the total number of support vectors retrieved during the training process. To define support vectors among available input vectors, the parameters $\alpha_{i, o}$ are used. The linearly nonseparable input data are transformed into a high-dimensional space, using $\vartheta(c)$ mapping function that allows classifying the data using linear hyperplane.

$f(c)=\operatorname{sign}\left[\sum_{i=1}^{P} \alpha_{i, o}\left(\vartheta(c) \vartheta\left(c_{i}\right)\right)+x\right]$
Table 3 Inner-product kernel function

\begin{tabular}{ll}
\hline Type & $S\left(\mathrm{c}, \mathrm{c}_{\mathrm{i}}\right), i=1,2,3, \ldots P$ \\
\hline Linear & $c^{T} c_{i}+x$ \\
Quadratic & $\left(x^{T} x_{i}+c\right)^{2}$ \\
Radial-basis function $(\mathrm{RBF})$ & $e^{\left(\frac{-\left.|c-| c_{i}\right|^{2}}{2 \sigma^{2}}\right)}$ \\
\hline
\end{tabular}

In order to minimize the complexity of optimization in higher dimensional feature space, we use the inner-product kernel function $S(c, z)=\vartheta(c) \vartheta(z)$. The updated decision the function can then be described as:

$f(c)=\operatorname{sign}\left[\sum_{i=1}^{P} \alpha_{i, o}\left(S\left(c, c_{i}\right)\right)+x\right]$

There are many kernel functions available that are used in support vector machine for classifying non-linear pattern such as linear kernels, string kernels, quadratic, radial basis kernels, polynomial etc. This work uses the linear kernel function, the quadratic kernel function and radial basis kernel, which are mathematically written as in Table 3. We have adopted the one-versus-rest approach for multi-class classification using SVM (Haider et al. 2018).

\subsubsection{K-nearest neighbor algorithm}

The KNN algorithm is low computational data classification technique, which requires $k$ nearest values for training samples in a particular feature space. The algorithm classifies a given dataset by based on majority vote using neighboring values. The particular data points are assigned to a specific data class which is common in the $k$ neighbor identified by the distance function. The distance function used in this paper is Euclidean and is given as:

Euclidean $=\sqrt{\sum_{i=1}^{k}\left(x_{i}-y_{i}\right)^{2}}$ 


\subsubsection{Random forest algorithm}

The RF algorithm for data classification is a combination of simple but various predictors, which reduces the computational complexity with great ease and achieves better results (Zhu et al. 2018) The RF algorithm is primarily modified version Bagging since it employs the regression tree process as the training algorithm (Shevchik et al. 2017). In RF algorithm, the selection of a random set of features develops a tree on the new training data points at each node to classify the split. The random forest algorithms lessen the computational cost in following ways: (1) the parallel ensemble, parallel computing process and the establishment of sub-models at each subset with no communication from the central processing unit (CPU) side. The introduction of sub-models developed on the newly established subsets reduces the training samples by a great extent. The replications of bootstraps, for instance, sample subsets, are less computationally expensive. Also, the learning procedure in the random forest algorithm of sub-models is said to be the regression tree.

\section{Results and discussion}

This section presents the experimental design, evaluation of data results obtained using S-Band sensing, discussion of features extracted using three machine learning algorithms such as SVM, KNN, and RF.

\subsection{Experimental setup}

The measurement and data acquisition has been carried out at School of Electronics Engineering, Xidian University, an indoor environment that replicates home or hospital scenario as shown in Fig. 1. The design consists of a 2024 IFR RF generator manufactured by ValueTronics connected to an omni-directional antenna that works as a transmitter. A PXIe-5630 vector network analyzer manufactured by National Instruments is connected to the four beam patch antenna that works as a receiver. The RF generator used here transmits ten packets per second.

Each packet contains a group of 30 frequency carriers at $2.4 \mathrm{GHz}$ over a $20 \mathrm{MHz}$ bandwidth that carry the wireless channel information in the form of variances of amplitude and phase information (Fan et al. 2018; Chen and Chiu 2018) The four beam patch antenna is the receiver used that collects narrow beams resulting in improved noise suppression and enabling multipath fading reduction. In the first part of the experiment as shown in Fig. 1a, microwave absorbing material manufactured by PN 2238 Liard Technologies (50 in total) is used to reduce the multipath fading in along the walls and see its impact on accuracy level of the system. In the second part as in Fig. 1b, the experiment was performed without deploying microwave absorbing material. The idea of using microwave absorbing material was to see the impact of multipath propagation (Yang et al. 2018a, b, c). It is observed (as discussed in a subsequent section) that the deployment of microwave absorbing material provided slightly better results but did not significantly improved the results of the performance parameters..

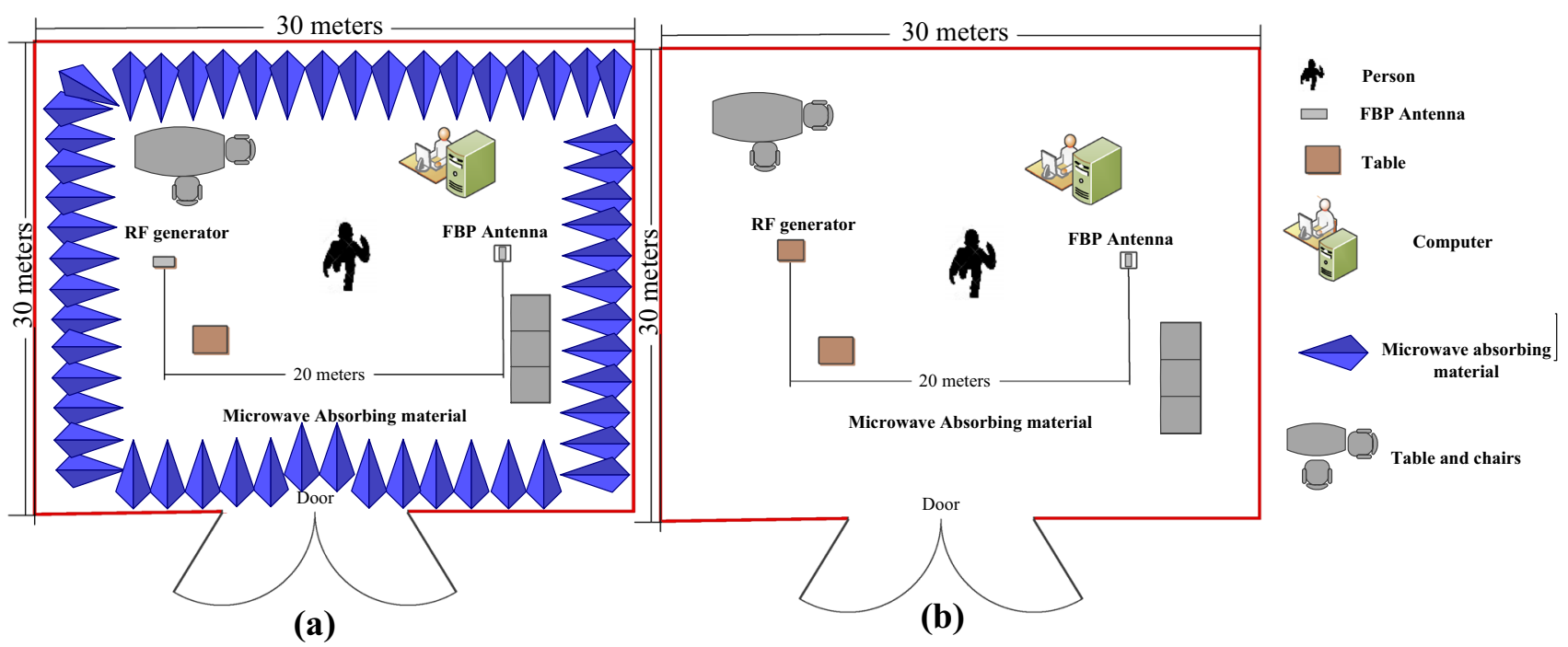

Fig. 1 Experimental setup for identifying various human activities for detecting epileptic seizure episodes. a Experimental design with the microwave absorbing material. b Experimental design without microwave absorbing material 
Table 4 Physical attributes of the human subjects

\begin{tabular}{lll}
\hline Subject \# & Weight $(\mathrm{kg})$ & Height $(\mathrm{cm})$ \\
\hline 1 & 69 & 161 \\
2 & 71 & 165 \\
3 & 71.5 & 164 \\
4 & 75 & 169 \\
5 & 75 & 169 \\
6 & 79 & 175 \\
7 & 80 & 168 \\
8 & 83 & 169 \\
9 & 85 & 171 \\
10 & 90 & 170 \\
\hline
\end{tabular}

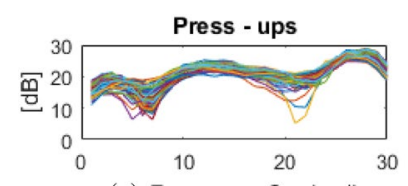

(a) Frequency Carrier \#

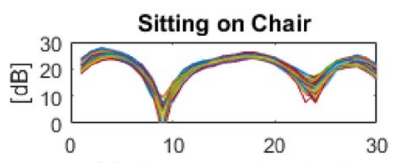

(c) Frequency Carrier \#

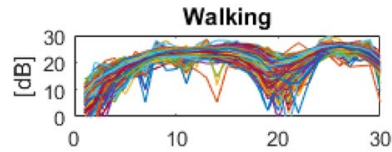

(b) Frequency Carrier \#

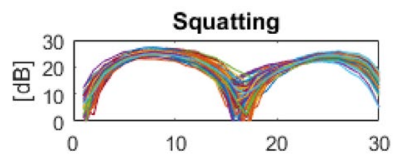

(d) Frequency Carrier \#

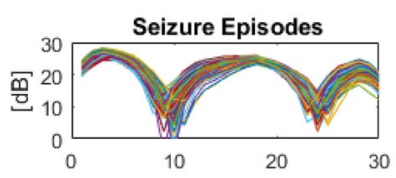

(e) Frequency Carrier \#

Fig. 2 The CFR obtained for five different human activities. a Pressups. b Walking. c Sitting-on-a-chair with slight body movements. d Squatting. e Epileptic seizure episodes

With the room dimensions of $30-\mathrm{m} \times 30-\mathrm{m}$ and ten human subjects participated in the experimental campaign. The physical attributes of the participants are presented in Table 4. The transmitter and receiver were placed $20 \mathrm{~m}$ apart and at a height of $0.5 \mathrm{~m}$. The proposed S-Band sensing technique continuously monitored the patients in an indoor environment. To characterize the seizure precisely from a clinical perspective and to differentiate other human activities that might be going around, we look at SVM, KNN and RF training, and classification. A detailed discussion is in part-B that highlights the overlap between the motions in the sensed signals. Hence, to compare the clinical versus normal conditions, we consider press-ups, walking, squatting along with the seemingly ideal situation of sitting.

\subsection{Measurement data}

The WCI data is measured in the S-Band. The sensing of every day human activities such as body movement or falls has been carried out. Specifically, we observe and measure epileptic seizures that are severe in which patient falls down and experiences involuntary body movement. The prime goal is to differentiate the variations in amplitude and phase with non-separable signals. As shown in Fig. 2, the perturbations as seen in amplitude are close in variations and hence the differentiating of clinical conditions is not accurate. Five different human activities have been analyzed for comparison using the channel frequency response (CFR) obtained using vector network analyzer, calibrated phase response and amplitude variations versus time history.

Figure 2 shows the channel frequency response results for five different activities in an indoor environment. The plots show CFR amplitude values as one of the indicators for detecting epileptic seizure episodes. In Fig. 2a when the subject was performing squatting activity, the variations in CFR amplitude changes accordingly. However, as the subject started walking the CFR amplitude information started varying significantly as seen in Fig. 2b. We observe the variances in CFR values as seen in Fig. 2c for an activity like sitting on a chair, when the subject was making small-scale body movements. With respect to sitting on the chair, we observe variations in CFR values in Fig. $2 \mathrm{~d}$ due to the press-up activity. Thus, we have listed the perturbations of the radio signals as acquired. The group of 30 subcarriers shows the total number of orthogonal frequency division multiplexing (OFDM) frequency channels received over a period of time. The OFDM over a $20 \mathrm{MHz}$ channel is made up of 64 subcarriers, out of which 48 are data carriers, 4 are pilot carriers and 12 as null subcarriers. The network interface card used only reports a group of 30 subcarriers as data carriers due to its intrinsic nature.

Examining the calibrated phase information for five activities, we observe that each human behavior perturbs the radio signal uniquely as seen in the WCI phase signal. It should be noted that the calibrated phase information is for all subcarriers. Comparatively, CFR signatures can be observed in the phase signals too. Clearly, we observe the phase variations are quite different, unlike amplitude. The numerical processing of this phase signal is complex. The phase signal as acquired by the vector network analyzer is visualized as a polar plot illustrated in Fig. 3. The calibrated phase information is analyzed to correlate with amplitude signal to improve the accuracy of the seizure detection as given by Eq. (11). In Fig. 3, green and red dots show the WCI phase data for regular and specific movements under seizure respectively. Figure 3 a shows calibrated phase information when the subject was doing press-ups activity on the ground. 
Fig. 3 Random and calibrated phase information obtained using S-Band sensing technique. a Press ups. b Walking. c Sitting-on-a-chair with slight body movements. $\mathbf{d}$ Squatting. $\mathrm{e}$ Epileptic seizure episodes

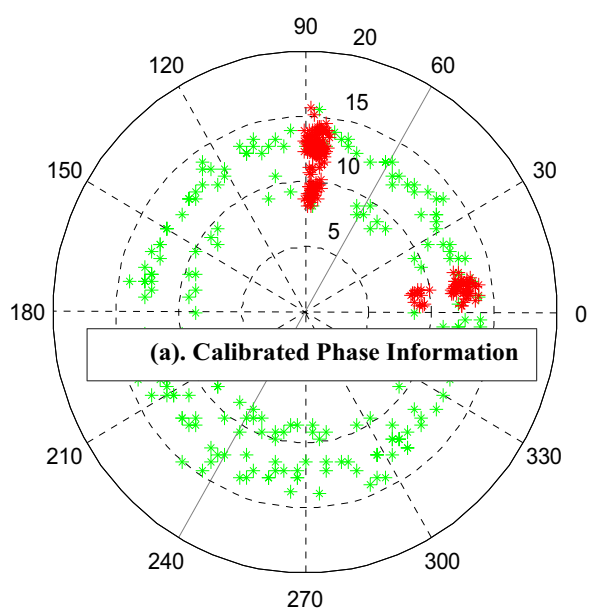

(a) Press - ups

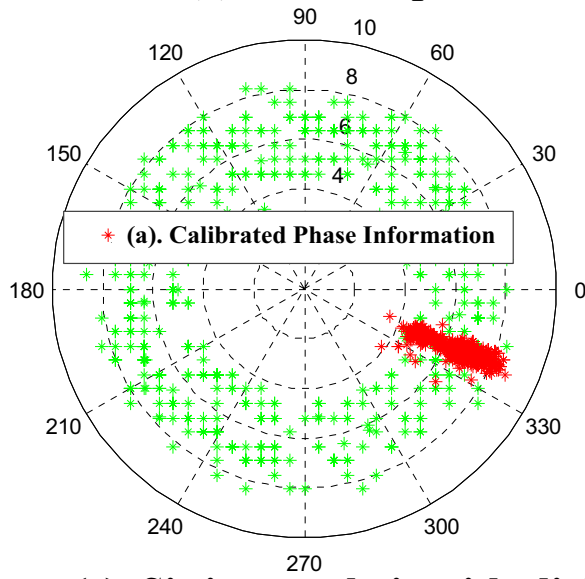

(c) Sitting on chair with slight movements

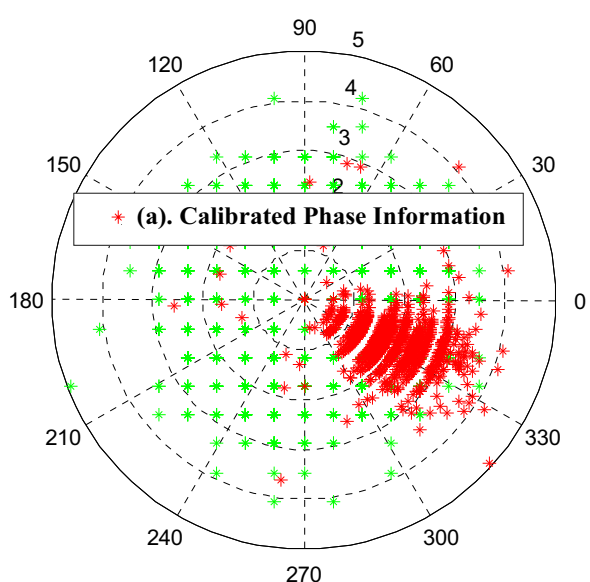

(b) Walking

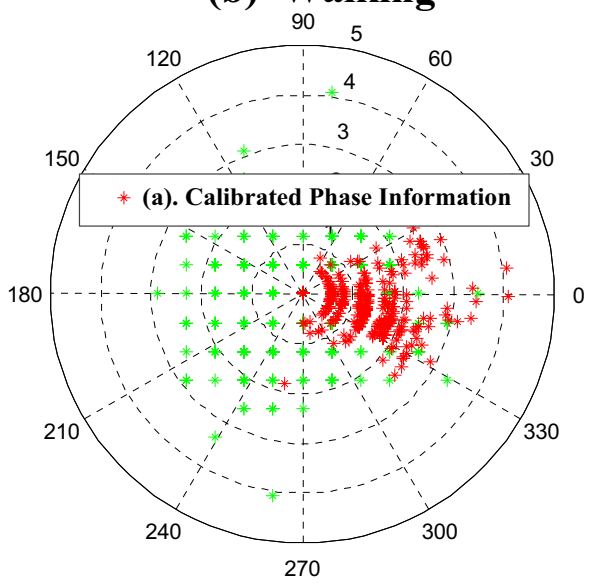

(d) Squatting

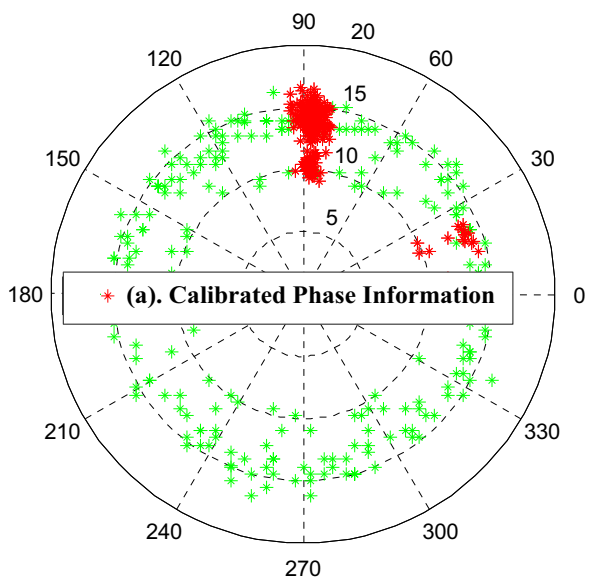

(e) Epileptic Seizure Episodes

However, when the subject was walking in the room, a large amount of scattered data can be seen in Fig. 3b. Activity such as sitting-on-a-chair as seen Fig. 3c plot is differentiable when compared to other moving activities. Moreover, the subject performing squatting activity, relative scattered data can be observed in Fig. 3d. In Fig. 3e, we show the phase values for an epileptic that is distinctive.

We further examine the time history of various activities as shown in Fig. 4, illustrating the overall time history of the subject carrying out various activities that is acquired over a total number of 2500 packets. The 


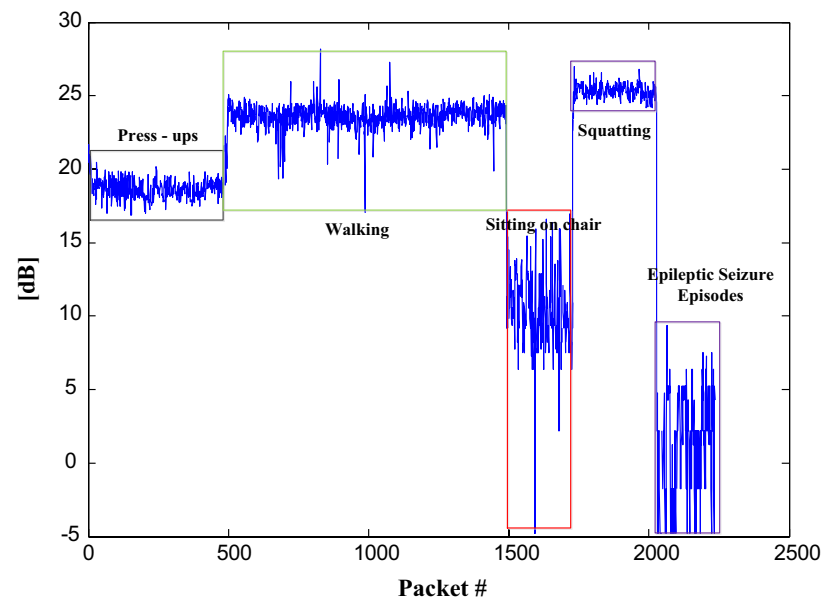

Fig. 4 Time history of five different activities considering frequency carrier \# 9

correlation of time and packet size is an empirical relation given as, for ten WCI packets received per second, implies

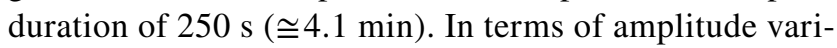
ations, we see the power level fluctuations around $10 \mathrm{~dB}$ from 0 to 500 packets $(\cong 50 \mathrm{~s})$ when the subject was performing press-ups activity. For walking activity, the amplitude level varies between 17 and $27 \mathrm{~dB}$ and we observe the fluctuation in 500th to 1500th packet ( $\cong 100 \mathrm{~s}$ ). However, when the subject was performing sitting-on-a-chair activity, power level fluctuates between -4 and $15 \mathrm{~dB}$ at 236 packets $(\cong 23.6 \mathrm{~s})$. Moreover, when squatting activity was performed, the power level fluctuated around $26 \mathrm{~dB}$ from 1736th to 2000 th packet ( $\cong 26.4$ s). Finally, when the subject fell on the ground experiencing epileptic seizure episodes, the power level started varying between -5 and $8 \mathrm{~dB}$ from 200 th to 2250 packet ( $\cong 22.5 \mathrm{~s}$ ).

Looking at ninth frequency carrier as in Fig. 4, we are able to differentiate the activities levels in the packets as the amplitude changes. The advantage of using wireless channel data is that we can choose on or multiple subcarriers for desired purpose. In this case, the subcarrier 9 presented the best results considering different human activites. This amplitude information versus time history clearly indicates unique power level for certain human activity. But there are instances where the power levels are same, for example, the power levels of press-ups activity and walking activity at 1000th the packet are similar. The power level $-4 \mathrm{~dB}$ at 1600th packet for sitting-on-a-chair activity and epileptic seizure episodes are same as well. The time history makes it difficult to achieve high classification accuracy in certain instances. Therefore, we use different machine learning algorithms such as SVM, KNN, and RF to classify various human activities with improved performance parameters such as accuracy, precision, specificity, recall,
Cohen's Kappa coefficient and F-a measure to detect seizure episodes.

\subsection{Feature extraction}

As discussed in Sect. 3, we used various machine learning algorithms to detect the seizure episodes. We first discuss the accuracy rate obtained using SVM algorithm leveraging a set of parameters called "features" that assist in creating a linear or non-linear classifier. Creation of SVM forms a basis for pattern recognition that depends on the selected features. In SVM, the basis set used are amplitude values from the training set as shown in Fig. 2. To implement the one-versusrest approach, we choose the samples of each class randomly from the $200 \mathrm{CFR}$ amplitudes. The features extracted from the signals as listed in Table 2. Data acquisition or sensing of the amplitude of the signal over a time interval in terms of a number of packets is done. For consistency, we have chosen amplitudes for feature extraction as it is non-separable with linearity between the activities. We then extract the features as a secondary set parameter for comparing the normal activities versus the epileptic seizures. The new set of features provides clear decision boundaries in the $\mathbb{R}^{2}$ space. Training dataset for a fixed number of classes with a number of features five and ten per class is for benchmarking purposes. The metrics for comparison is based on usage of kernel function listed in Table 3. The testing data is transformed using the same set of kernel methods as a measure to predict and classify them.

Using a kernel function allows the decision boundaries in a higher dimensional feature space. The functions provide dot product or inner products to feature set that take care of non-separable feature set. The polynomial kernel function is simple and easy to compute. The radial basis function provides a bell-curve with a variability adjustment in the feature set $(\sigma)$.

\subsection{Data classification using SVM}

This section deals with the discussion of results obtained using SVM considering the percentage accuracy only. The remaining four performance parameters such as precision, recall, Kappa, and F-measure are discussed in later section. We first consider the data obtained when microwave absorbing material was used. The data in Fig. 2 that is variations of amplitude information against group of 30 subcarriers is used to classify the data using different machine algorithms. To differentiate the activities clinical or generic movements, we perform training with four classes of activities namely press-ups, walking, sitting and squatting. To compare these with seizure we look at a finite number of samples in the training phase and classify with the testing data. We have used svmtrain, built-in function in MATLAB to train the 
Table 5 Accuracy rate obtained using SVM for seizure detection $(\%)$

\begin{tabular}{|c|c|c|c|c|c|c|c|c|c|c|c|}
\hline \multirow{2}{*}{$\begin{array}{l}\text { Kernel } \\
\text { Function }\end{array}$} & \multicolumn{6}{|c|}{ Five features } & \multicolumn{5}{|c|}{ Ten features } \\
\hline & $\mathrm{S}$ & 1 & 2 & 3 & 4 & 5 & 1 & 2 & 3 & 4 & 5 \\
\hline \multicolumn{12}{|c|}{ (a) Accuracy rate obtained using SVM for seizure detection using absorbing material } \\
\hline \multirow[t]{3}{*}{ Linear } & 40 & 95.75 & 61.25 & 86.00 & 82.75 & 55.50 & 98.25 & 75.50 & 83.00 & 91.75 & 69.00 \\
\hline & 80 & 98.00 & 61.00 & 85.75 & 82.50 & 69.50 & 98.50 & 78.75 & 84.25 & 93.25 & 76.00 \\
\hline & 120 & 98.25 & 65.75 & 86.00 & 83.25 & 73.50 & 98.75 & 82.25 & 83.50 & 93.50 & 83.75 \\
\hline \multirow[t]{3}{*}{ Quadratic } & 40 & 87.50 & 73.00 & 88.00 & 85.00 & 74.50 & 96.50 & 82.00 & 85.75 & 90.00 & 89.75 \\
\hline & 80 & 94.25 & 69.50 & 88.25 & 82.50 & 84.75 & 98.25 & 82.25 & 86.25 & 93.75 & 93.50 \\
\hline & 120 & 97.50 & 72.50 & 88.75 & 85.25 & 86.75 & 98.75 & 82.25 & 89.50 & 94.00 & 96.00 \\
\hline \multirow[t]{3}{*}{$\mathrm{RBF}$} & 40 & 91.50 & 64.25 & 89.25 & 89.50 & 75.75 & 96.50 & 80.00 & 88.25 & 92.00 & 88.00 \\
\hline & 80 & 93.75 & 69.25 & 87.50 & 83.75 & 83.75 & 97.75 & 79.25 & 89.00 & 95.00 & 92.50 \\
\hline & 120 & 96.00 & 72.50 & 89.25 & 86.00 & 86.50 & 98.75 & 92.75 & 91.75 & 94.25 & 95.25 \\
\hline
\end{tabular}

(b) Accuracy rate obtained using SVM for seizure detection without using absorbing material

\begin{tabular}{lrllllllllll} 
Linear & 40 & 89.23 & 58.32 & 83.47 & 80.56 & 53.47 & 95.74 & 70.17 & 80.00 & 91.64 & 67.42 \\
& 80 & 96.87 & 60.78 & 83.17 & 79.00 & 93.38 & 95.04 & 76.00 & 81.64 & 92.11 & 74.95 \\
& 120 & 85.45 & 66.87 & 85.64 & 79.45 & 71.74 & 97.00 & 78.54 & 80.50 & 89.51 & 81.00 \\
Quadratic & 40 & 92.00 & 67.85 & 88.72 & 70.00 & 68.94 & 93.50 & 79.84 & 80.00 & 88.94 & 85.10 \\
& 80 & 91.57 & 68.45 & 85.97 & 79.37 & 80.00 & 95.28 & 79.80 & 81.37 & 89.00 & 90.29 \\
& 120 & 94.67 & 70.50 & 86.42 & 82.38 & 93.54 & 97.14 & 81.14 & 88.97 & 87.93 & 91.74 \\
RBF & 40 & 88.97 & 60.54 & 85.78 & 86.47 & 71.57 & 95.00 & 79.47 & 90.47 & 90.78 & 84.47 \\
& 80 & 90.54 & 61.98 & 84.78 & 80.64 & 80.00 & 94.78 & 75.67 & 86.78 & 91.00 & 88.97 \\
& 120 & 91.00 & 70.58 & 87.54 & 85.14 & 98.00 & 96.54 & 80.00 & 90.87 & 90.11 & 91.78 \\
\hline
\end{tabular}

SVM classifier for classifying different human activities or classes. With the inbuilt sequential minimum optimization solver, we obtain the optimal decision boundary. Table 5a shows the five classes $\{1,2,3,4,5\}$ for the activities namely press-ups, walking, sitting-on-a-chair, squatting and seizure per feature set as listed in Table 1. It should be noted that the Table 5a illustrates the results obtained when microwave absorbing material was used. The size of data samples in the training set, $S=\{40,80,120\}$ for each of the kernel functions.

The sample size is 30 unique signals pertaining to the 30 subcarriers. Out of 240 samples, the first 40,80 and 120 samples from each class were used for training, while the last 120 samples of each class were used for testing our SVM. Inherent to the SVM created, with our linear classifiers to minimize the empirical risk of misclassification. This risk factor is used in the calculation of our accuracy rate that is averaged for all the 120 samples per class. For each class was used to train the SVM for two sets of features, one considering 5 features per class and other with all the ten features.

We have set scaling factor $\sigma=1$ for RBF kernel function and order of $\mathrm{p}=3$ for quadratic kernel function. The selectivity of features as a subset versus a larger set of features increases the unique characteristics of the signal is compared in Table 5.

First, we discussed the accuracy rate obtained as shown in Table 5a using SVM when microwave absorbing material was used. The percentage accuracy for press-ups, walking, sitting, squatting and epileptic seizure episodes are 95.75, $61.25,86.00,82.75$ and 55.55 , respectively when 40 training samples were used considering five SVM features by applying linear kernel function. However, the accuracy rate improved when ten SVM features as described in Table 2 were used. For example, the percentage of more than $90 \%$ was achieved for press-up and squatting activities. The epileptic seizure episodes receive an accuracy of $69.00 \%$ in this case. Slight improvement was observed when 80 training samples were used considering five SVM features by applying linear kernel function. The accuracy rate of $98.00 \%$, $61.00 \%, 85.75 \%$, and $69.60 \%$ was obtained for press-ups, walking, sitting, squatting and seizure episodes, respectively.

However, there were marginal improvements in results obtained for ten SVM features. For example, the accuracy rate of walking activity improved by $17 \%$, squatting activity improved by nearly $11 \%$ and epileptic seizure episodes by $6.5 \%$. However, the results obtained for 120 training samples considering five SVM features were almost identical to the ones which used 80 SVM features. When ten SVM features were used for 120 training samples by applying linear kernel function, better results were obtained. For examples, the two activities such as press-ups and squatting received the percentage accuracy of $98.75 \%$ and $93.50 \%$, respectively. The other three activities including the epileptic seizure episodes, an accuracy of nearly $83 \%$ was obtained. The quadratic kernel function performed slightly better than linear 
kernel function. The accuracy rate for press-ups, walking, sitting, squatting and seizure episodes was $87.51 \%, 73.00 \%$, $88.00 \%, 85.00 \%$, and $74.50 \%$, respectively when 40 training samples were used for five SVM features. When ten SVM features were used for similar kernel function, each activity received an accuracy of more than $80 \%$. The epileptic seizure episodes received an accuracy of $89.75 \%$. There was slight variation observed in the results obtained using 80 training samples. For example, the accuracy of seizure episodes improved by almost $10 \%$.

The accuracy rate for quadratic kernel function considering five features was more than $80 \%$ for four activities except for walking activity which was $72.50 \%$. The seizure episodes received an accuracy of $86.75 \%$. When ten SVM features were used, three activities such as press-ups, squatting and seizure episodes received percentage accuracy of more than $90 \%$. While walking activity and sitting activity obtained accuracy are of $82.24 \%$ and $89.50 \%$, respectively. The results obtained for five activities considering five SVM features for 40, 80 and 120 training samples are almost identical with slight variations in the values. It can be seen that the RBF kernel function obtained percentage accuracy of more than $90 \%$ for each human activity. The epileptic seizure episodes received an accuracy of $94.25 \%$.

Looking at Table 5b, which contains the results, obtained when no microwave absorbing materials were used. The percentage accuracy decreased by nearly $4-6 \%$ for each human activity when various kernel function and different SVM features were considered. It implies that there is a marginal decrease in accuracy rate. Hence we use the results obtained using ten SVM features and RBF kernel when no microwave absorbing materials were deployed inside the room.

\subsection{Data classification using KNN and RF}

The results obtained using the proposed method was processed the other two machine learning algorithms such as KNN and RF. The KNN data classification technique is a supervised learning method that classified the data based voting criteria. The objects closest to the $k$ values from the training data points considered assign a new object to a certain class on the basis of majority votes. The training process involves in KNN algorithm comprise features storing and labeling the classes of the certain training data points (Liu et al. 2018). During classification process, the most recurrent training data samples $k$ appoint an unlabeled object of a certain data class. Different distance metrics are used in KNN data classification technique. In this paper, Euclidean distance as in Eq. (11) has been used and the value of $k$ is set to 1 that means the selected data class label was similar to the one closes to the training data. Moreover, the random forest data classification technique integrates a set of independent decision tree classifier (Breiman 2001). The decision tree in random forest algorithm has $N$ number of leaves break downs the feature space into $N$ regions such as $Z_{n}, 1 \leq m \leq R$. The prediction function $f(x)$ for each tree is described as:

$f(x)=\sum_{n=1}^{R} c_{n} \Re\left(x, Z_{n}\right)$

where $R$ denotes the number of regions in a feature space, $Z_{n}$ is $n$th region, $c_{n}$ denotes a constant value which corresponds to $n$, and $\Re$ is termed as the indicator function.

$\mathfrak{R}\left(x, Z_{n}\right)=\left(\begin{array}{l}1, \text { if } x \varepsilon Z_{n} \\ 0, \text { otherwise }\end{array}\right.$

The decision function is based on majority vote of all the trees in the random forest algorithm (Quek et al. 2016). In this paper, the RF algorithm with 100 trees is used. Furthermore, the five performance parameters accuracy, precision, recall, kappa and F-measure used in three machine learning algorithms are discussed below. The primary reason for selecting other performance metrics is that accuracy works best when the data set obtained is balanced. However, it fails to provide adequate results when data is imbalanced, employing that the available dataset for each class is equal in number. That is why we have used other performance measures to evaluate the results obtained of proposed system.

Accuracy $=\frac{T p+T n}{F p+F n+T p+T n}$

Accuracy is termed as a portion of instances correctly classified. Here Tp is true positive, Tn is true negative, Fp is false positive and $\mathrm{Fn}$ is false negative.

Precision $=\frac{T p}{T p+F p}$

Precision is the portion of relevant instances among the retrieved instances.

Recall $=\frac{T p}{T p+F n}$

Recall is the portion of the relevant instances successfully retrieved

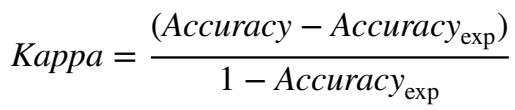

Kappa is the actual accuracy to the expected accuracy of a system

$F$-measure $=\frac{2 T p}{(F n+F p+2 T p)}$

F-measure is the harmonic mean of precision and recall. 
Table 6 Confusion matrix obtained for various activities using different algorithms

Press-ups Walking Sitting Squatting Seizures

(a) Confusion matrix obtained for various activities using SVM

$\begin{array}{lrrrrr}\text { Press-ups } & 116 & 9 & 4 & 2 & 2 \\ \text { Walking } & 1 & 98 & 1 & 2 & 2 \\ \text { Sitting } & 1 & 8 & 112 & 1 & 3 \\ \text { Squatting } & 1 & 3 & 2 & 112 & 4 \\ \text { Seizures } & 1 & 2 & 3 & 5 & 111\end{array}$

(b) Confusion matrix obtained for various activities using KNN

$\begin{array}{lrrrrr}\text { Press-ups } & 101 & 4 & 3 & 8 & 5 \\ \text { Walking } & 7 & 96 & 4 & 7 & 4 \\ \text { Sitting } & 3 & 8 & 109 & 9 & 5 \\ \text { Squatting } & 5 & 3 & 3 & 88 & 5 \\ \text { Seizures } & 4 & 7 & 2 & 7 & 101\end{array}$

(c) Confusion matrix obtained for various activities using RF

\begin{tabular}{lrrrrr} 
Press-ups & 95 & 8 & 2 & 3 & 2 \\
Walking & 5 & 103 & 3 & 5 & 1 \\
Sitting & 4 & 8 & 111 & 1 & 2 \\
Squatting & 3 & 3 & 2 & 89 & 2 \\
Seizures & 3 & 5 & 1 & 2 & 103 \\
\hline
\end{tabular}

\subsection{Results obtained using SVM, KNN and RF algorithm}

The K- nearest neighbor and random forest algorithms were trained and tested using 120 samples. The confusion matrices obtained for the three machine learning algorithms are presented in Table 6 . Table 7 shows the value of five performance values obtained for five human activities such as press-ups, walking, sitting, squatting and seizure episodes.

Table 7a indicates the values received using support vector machine. The percentage accuracy of four human activities is more than $90 \%$ and $80 \%$ for walking activity. The precision values for walking, sitting, squatting and seizure episodes are more than $90 \%$ except for press-ups activity which is $85.92 \%$. The recall values for press-ups, walking, sitting and squatting vary between $85 \%$ and $89 \%$. The recall value for epileptic seizure episode is $92.5 \%$. The F-measure values for press-ups, sitting and seizure episodes are more than $90 \%$ and approximately $88 \%$ for walking and squatting. The overall percentage accuracy for SVM algorithm considering five human activities is 89.86 and overall Cohen's Kappa coefficient $87 \%$.

As far as the values of five performance parameters are concerned when KNN algorithm was used, values obtained are presented in Table $7 \mathrm{~b}$. One activity received an accuracy of $90 \%$, three activities such as press-ups, walking, and seizures obtained accuracy around $84 \%$. The precision and recall values obtained vary between $81 \%$ and $84 \%$. The press-ups activity, sitting activity, and seizure episodes received F-measure values around $84 \%$. F-measure value for squatting activity is $78.9 \%$ and $68 \%$ for walking activity. Table $7 \mathrm{c}$ shows the values of five performance parameters obtained using random forest algorithm.

The accuracy rate for press-ups, walking, sitting, squatting and seizure episodes is $79.16 \%, 85.83 \%, 92.50 \%$, $74.16 \%$ and $85.82 \%$, respectively. The recall values for RF algorithm vary between 80 and $84 \%$. The recall values vary between $81 \%$ and $84 \%$ for four human activities except for seizure episodes which is $91.6 \%$. The F-measure
Table 7 Classification results obtained using different algorithms

\begin{tabular}{|c|c|c|c|c|c|c|}
\hline & Accuracy & Precision & Recall & Overall Acc & F-measure & Kappa \\
\hline \multicolumn{7}{|c|}{ (a) Classification results obtained using SVM (\%) } \\
\hline Press-ups & 96.54 & 85.92 & 85.9 & \multirow[t]{5}{*}{89.86} & 90.9 & \multirow[t]{5}{*}{0.879} \\
\hline Walking & 80.00 & 93.33 & 87.5 & & 88.3 & \\
\hline Sitting & 90.87 & 89.60 & 86.8 & & 93.2 & \\
\hline Squatting & 90.11 & 92.50 & 89.6 & & 88.5 & \\
\hline Seizures & 91.78 & 90.98 & 92.5 & & 91.4 & \\
\hline \multicolumn{7}{|c|}{ (b) Classification results obtained using KNN (\%) } \\
\hline Press-ups & 84.16 & 83.47 & 83.5 & \multirow[t]{5}{*}{82.8} & 83.8 & \multirow[t]{5}{*}{0.786} \\
\hline Walking & 81.35 & 81.35 & 81.1 & & 68.0 & \\
\hline Sitting & 90.80 & 81.34 & 81.3 & & 85.8 & \\
\hline Squatting & 73.95 & 84.60 & 84.6 & & 78.9 & \\
\hline Seizures & 84.16 & 84.10 & 84.1 & & 84.1 & \\
\hline \multicolumn{7}{|c|}{ (c) Classification results obtained using RF (\%) } \\
\hline Press-ups & 79.16 & 84.07 & 84.0 & \multirow[t]{5}{*}{83.9} & 85.2 & \multirow[t]{5}{*}{0.794} \\
\hline Walking & 85.83 & 80.00 & 83.1 & & 84.5 & \\
\hline Sitting & 92.50 & 81.60 & 81.9 & & 86.3 & \\
\hline Squatting & 74.16 & 84.12 & 84.1 & & 79.2 & \\
\hline Seizures & 85.83 & 84.34 & 91.6 & & 85.1 & \\
\hline
\end{tabular}


values received are almost $85 \%$ for four human activities except for squatting activity which is $78.9 \%$. The overall accuracy obtained using RF algorithm is $83.94 \%$ and overall Cohen's Kappa coefficient 79.4\%.

By examining all the values obtained using three machine learning algorithms, we notice decrease in values for five performance parameters when KNN and RF algorithm were used. Hence, we conclude that the SVM classifier performed better than KNN and RF algorithms when classifying the aforementioned five human activities.

\section{Conclusion}

The proposed system exploited different devices such as vector network analyzer, RF generator, omni-directional antenna, four beam patch antenna, etc. to obtain the wireless channel measurements known as wireless channel information. The aim was monitoring epileptic patients and differentiating epileptic seizure episodes with respect to other activities. Systematically, we consider the training phase that is corroborated by our raw data analysis in terms of amplitude and phase of the received signal. Statistically, we compute a feature set that measures the degree of variance and characteristics of the raw data obtained using S-Band sensing. Using different machine learning algorithms such as SVM, KNN, and RF, we classify data by training the models of each classifier with a dataset. By examining the various performance parameters (accuracy, precision, recall, Kappa and F-measure) of the three classifiers, it was observed that the SVM algorithm delivered best results.

To improve the performance of the system, sanitized phase information was used and a directive four beam patch antenna is employed to mitigate multipath propagation errors making this work ideal for an indoor environment. The WCI data collectively detect the epileptic seizure episodes based on samples used as training and testing data. The system was tested on ten different subjects for five human activies. However, the proposed solution would work for more or less activities since the system is a generalized one where data can be captured for any activity and the machine learning algorithms can classify as many activities as provided. The proposed method can be used as drug monitoring and therapeutic facilities for patients that suffer from chronic seizure episodes. The prime purpose is for documenting the patient's health record that aid in giving a better quality of life.

Funding The work was supported in part by the Fundamental Research Funds for the Central Universities (No. JB180205), International Scientific and Technological Cooperation and Exchange Projects in Shaanxi Province (No. 2017KW-005), and China Postdoctoral Science Foundation Funded Project (No. 2018T111023).

\section{References}

Abdelnasser H, Youssef M, Harras KA (2014) WiGest: a ubiquitous WiFi-based gesture recognition system. In: Proc. IEEE INFOCOM, Piscataway, New Jersey, US

Abdelnasser H, Youssef M, Harras KA (2015) WiGest: a ubiquitous WiFi-based gesture recognition system. In: Proc. IEEE INFOCOM

Alemdar H, Ersoy C (2017) Smart environment architecture for robust people detection by infrared and visible video fusion. J Ambient Intell Human Comput 8:513. https://doi.org/10.1007/s1265 2-016-0440-x

Azimi I, Rahmani AM, Liljeberg P et al (2017) Internet of things for remote elderly monitoring: a study from user-centered perspective. J Ambient Intell Human Comput 8:273. https://doi. org/10.1007/s12652-016-0387-y

Ben-Arie J, Wang Z, Pandit P, Rajaram S (2002) Human activity recognition using multidimensional indexing. IEEE Trans Pattern Anal Mach Intell 24(8):1091-1104

Ben-Arie J, Wang Z, Pandit P, Rajaram S (2012) Human activity recognition using multidimensional indexing. IEEE Trans Pattern Anal Mach Intell 24(8):1091-1104

Breiman L (2001) Random forests. J Mach Learn 45:5-32

Castillo JC, Fernández-Caballero A, Serrano-Cuerda J et al (2017) Smart environment architecture for robust people detection by infrared and visible video fusion. J Ambient Intell Human Comput 8:223. https://doi.org/10.1007/s12652-016-0429-5

Chen T, Chiu MCJ (2018) Smart technologies for assisting the life quality of persons in a mobile environment: a review. Ambient Intell Human Comput 9:319. https://doi.org/10.1007/s1265 2-016-0396-x

Dilber D, Kaur J (2016) EEG-based detection of epilepsy by a mixed design approach. In: IEEE international conference on recent trends in electronics, information and communication technology (RTEICT), Bangalore, pp 1425-1428

Fan D et al (2018) Breathing rhythm analysis in body centric networks. IEEE Access 6:32507-32513. https://doi.org/10.1109/ ACCESS.2018.2846605

Giorgetti G, Cidronali A, Gupta SKS, Manes G (2007) Exploiting low-cost directional antennas in 2.4 GHz IEEE 802.15.4 wireless sensor networks. In: European conference on wireless technologies, Munich, pp 217-220

Haider D, Ren A, Fan D et al (2018) Utilizing a 5G spectrum for health care to detect the tremors and breathing activity for multiple sclerosis. Trans Emerg Tel Tech 29:e3454. https://doi. org/10.1002/ett.3454

Liu L, Shah SA, Zhao G, Yang X (2018) Respiration symptoms monitoring in body area networks. Appl Sci 8:568

Lotfi A, Langensiepen C, Mahmoud SM et al (2012) Smart homes for the elderly dementia sufferers: identification and prediction of abnormal behavior. J Ambient Intell Human Comput 3:205. https://doi.org/10.1007/s12652-010-0043-x

Pedram MZ, Shamloo A, Alasty A, Ghafar-Zadeh E (2015) MRIguided epilepsy detection. In: 2015 37th annual international conference of the IEEE engineering in medicine and biology society (EMBC), Milan, pp 4001-4004

Quek YT, Woo WL, Logenthiran T (2016) DC equipment identification using K-means clustering and kNN classification techniques. In: IEEE region 10 conference (TENCON), Singapore, pp 777-780. https://doi.org/10.1109/TENCON.2016.7848109

Shah SA et al (2016) Posture recognition to prevent bedsores for multiple patients using leaking coaxial cable. IEEE Access 4:8065-8072

Shevchik SA, Saeidi F, Meylan B, Wasmer K (2017) Prediction of failure in lubricated surfaces using acoustic time-frequency 
features and random forest algorithm. IEEE Trans Ind Inf 13(4):1541-1553. https://doi.org/10.1109/TII.2016.263508

Sigg S, Scholz M, Shi S, Ji Y, Beigl M (2014) RF-sensing of activities from non-cooperative subjects in device-free recognition systems using ambient and local signals. IEEE Trans Mobile Comput 13(4):907-920

Song B, Kamal AT, Soto C, Ding C, Farrell JA, Roy-Chowdhury AK (2010) Tracking and activity recognition through consensus in distributed camera networks. IEEE Trans Image Process 19(10):2564-2579

Tambe SB, Gajre et al (2018) Cluster-based real-time analysis of mobile healthcare application for prediction of physiological data. J Ambient Intell Human Comput 9:429. https://doi. org/10.1007/s12652-017-0562-9

Tao D, Jin L, Wang Y, Li A (2014) Rank-preserving discriminant analysis for human behavior recognition on wireless sensor networks. IEEE Trans Ind Inf 10(1):813-823
Yang X et al (2018a) Freezing of gait detection considering leaky wave cable. IEEE Trans Antennas Propag. https://doi. org/10.1109/TAP.2018.2878081

Yang X et al (2018b) Detection of essential tremor at the S-band. IEEE J Transl Eng Health Med 6:1-7. https://doi.org/10.1109/ JTEHM.2017.2789298(Art no. 2000107)

Yang X et al (2018c) Monitoring of patients suffering from REM sleep behavior disorder. IEEE J Electromagn RF Microwaves Med Biol 2(2):138-143. https://doi.org/10.1109/JERM.2018.2827705

Zhu M et al (2018) Class weights random forest algorithm for processing class imbalanced medical data. IEEE Access 6:4641-4652. https://doi.org/10.1109/ACCESS.2018.2789428

Publisher's Note Springer Nature remains neutral with regard to jurisdictional claims in published maps and institutional affiliations. 